\title{
Effects of Delta-9-Tetrahydrocannabinol and Cannabidiol on Cisplatin-Induced Neuropathy in Mice
}

\author{
Authors \\ Hannah M. Harris ${ }^{1}$, Kenneth J. Sufka ${ }^{1,2,3}$, Waseem Gul ${ }^{3}$, Mahmoud A. ElSohly ${ }^{3,4}$ \\ Affiliations \\ ${ }^{1}$ Department of Psychology, University of Mississippi, University, MS, USA \\ ${ }^{2}$ Department of Pharmacology, University of Mississippi, University, MS, USA \\ ${ }^{3}$ National Center for Natural Products Research, University of Mississippi, University, MS, USA \\ ${ }^{4}$ Department of Pharmaceutics, University of Mississippi, University, MS, USA
}

Key words

- cisplatin-induced neuropathy

- delta-9-tetrahydrocannabinol

- cannabidiol

tactile allodynia

\section{Abstract}

$\nabla$

Sativex, a cannabinoid extract with a $1: 1$ ratio of tetrahydocannabinol and cannabidiol, has been shown to alleviate neuropathic pain associated with chemotherapy. This research examined whether tetrahydocannabinol or cannabidiol alone could attenuate or prevent cisplatin-induced tactile allodynia. In experiment 1 , mice $(\mathrm{C} 57 \mathrm{BL} / 6)$ received eight administrations of $2.3 \mathrm{mg} / \mathrm{kg}$ cisplatin or saline solution IP every other day to induce tactile allodynia. Mice were then administered vehicle, $100 \mathrm{mg} / \mathrm{kg}$ gabapentin, $2 \mathrm{mg} / \mathrm{kg}$ tetrahydocannabinol, or $2 \mathrm{mg} / \mathrm{kg}$ cannabidiol IP and tested 60 min later on an electronic Von Frey. In experiment 2, prevention studies, cannabidiol $(0.0,0.5,1.0$, and $2.0 \mathrm{mg} / \mathrm{kg})$ or tetrahydocannabinol $(0.0,0.5,1.0$, and $2.0 \mathrm{mg} / \mathrm{kg})$ was given IP 30 min prior to cisplatin administration (2.3 or $1.0 \mathrm{mg} / \mathrm{kg}$ ) utilizing a six-dose alternate day protocol. In both studies, tactile responses to the hind paws were quantified in $g$ of force using

\section{Introduction}

$\nabla$

revised March 30, 2016

accepted April 2, 2016

Bibliography

DOI http://dx.doi.org/

10.1055/s-0042-106303

Published online May 23, 2016

Planta Med 2016; 82:

1169-1172 @ Georg Thieme

Verlag KG Stuttgart · New York .

ISSN 0032-0943

Correspondence

\section{Hannah M. Harris}

Department of Psychology

University of Mississippi

101 B Peabody Building

University, MS 38677

USA

Phone: +16629155390

Fax: + 16629155398

hmharris@go.olemiss.edu
Pain is a common complaint in oncology patients and can diminish their quality of life $[1,2]$. Cisplatin is a common and effective chemotherapeutic against a variety of tumors. However, it has a dose-limiting effect, where $40-80 \%$ of patients develop neuropathic pain 3-6 months into treatment [3]. Moreover, CIN does not abate upon discontinuation of treatment, a phenomenon known as "coasting" [4]. A large meta-analysis revealed that CIN was prevalent in $68 \%, 60 \%$, and $30 \%$ of patients one, three, and greater than six months after cisplatin discontinuation, respectively [5]. Several approaches to prevent CIN have yielded disappointing results, as these compounds also interfere with cisplatin's antitumor properties [6, 7]. Current treatment for CIN includes nortrip- an electronic Von Frey prior to and after the cisplatin administration protocol. Cisplatin produced a reduction in $\mathrm{g}$ of force indicative of neuropathy that was attenuated by gabapentin, tetrahydocannabinol, and cannabidiol but not prevented by either cannabinoid. These data demonstrate that each of the major constituents of Sativex alone can achieve analgesic effects against cisplatin neuropathy.

\section{Abbreviations \\ $\nabla$ \\ CB: cannabinoid \\ CBD: cannabidiol \\ CIN: cisplatin-induced neuropathy \\ eVF: $\quad$ electronic Von Frey \\ IP: intraperitoneal \\ THC: $\quad \Delta$ 9-tetrahydrocannabinol}

Supporting information available online at http://www.thieme-connect.de/products

tyline, gabapentin, and lamotrigine. Each of these have shown efficacy against other neuropathic pain syndromes, but show little efficacy in CIN [8]. Collectively, these observations suggest a need to identify novel targets for the treatment of CIN.

Targeting the cannabinoid system has shown efficacy in a variety of clinical syndromes including pain. Synthetic cannabinoids produce analgesia in a number of nociceptive assays and these effects can be produced by targeting either CB1 and/or CB2 receptors [9-11]. There is also recent evidence that $C B$ targets may be efficacious in treating CIN. For example, selective CB1 and CB2 receptor agonists, as well as compounds that increase endocannabinoid tone, attenuate tactile allodynia in rodent models of CIN [12-14]. 
Reports indicate that cannabis products may be useful for a number of clinical syndromes [15]. The two major constituents in cannabis are THC and CBD, both of which affect CB1 and CB2 receptors, with CBD showing a lower affinity to each [16]. Sativex is a proprietary whole-plant cannabis extract that contains a $1: 1$ ratio of THC and CBD (2.7 mg: $2.5 \mathrm{mg}$ ). Sativex has been approved in Canada and the UK to treat multiple sclerosis, neuropathic pain, and opioid-resistant cancer pain $[17,18]$. How the major constituents in Sativex perform against CIN is unknown. It may be that THC or CBD administered alone is effective in alleviating or, perhaps, preventing CIN.

\section{Results}

$\nabla$

Experiment 1: The effects of cisplatin on tactile allodynia are summarized in $\odot$ Fig. 1. Paw withdrawal thresholds in cisplatin mice were lower than non-cisplatin mice and illustrate tactile allodynia associated with neuropathy. A one-way ANOVA on these data revealed a significant treatment effect $[F(1,29)=4.972$, $\mathrm{p}=0.034]$.

The effects of gabapentin, THC, and CBD on paw withdrawal thresholds across cisplatin treatment conditions are summarized in Fig. 2. In general, non-cisplatin-treated groups had higher paw withdrawal thresholds than cisplatin groups. In non-cisplatin-treated mice, gabapentin, THC, and CBD did not alter paw withdrawal thresholds. In the vehicle group, cisplatin produced tactile allodynia (i.e., lowered thresholds). This allodynic response was attenuated by all three of the test compounds. Consistent with these observations, a two-way ANOVA revealed a significant main effect for drug treatment $[F(3,23)=4.08, \mathrm{p}=0.018]$ and a significant Cisplatin by drug interaction term $[F(3,23)=3.39, \mathrm{p}=0.034]$. The main effect for Cisplatin treatment was not significant $[F(1,23)=1.99, p=0.17]$. These findings prompted a simple effects analysis for drug treatment in both the cisplatin and non-cisplatin groups; a main effect for drug treatment was found for the cisplatin groups $[F(3,12)=6.10$, $\mathrm{p}=0.009$ ], but not in the controls. Among the cisplatin groups, mean paw thresholds were significantly higher in gabapentin $(p=0.002)$, THC $(p=0.007)$, and CBD $(p=0.035)$-treated mice compared to saline mice.

Experiments $2 a$ and $b$ : The effects of THC and CBD in preventing cisplatin tactile allodynia are summarized in 0 Fig. 3. In both studies, cisplatin lowered paw withdrawal thresholds on day 12 from baseline measures taken on day 1 in the cisplatin administration protocol. Neither THC nor CBD, at any doses tested, affected the paw withdrawal thresholds. One-way ANOVAs on each data set failed to reveal significant main effects of either the THC or $\mathrm{CBD}$ dose ( $\mathrm{ps}=\mathrm{n}$. s.). No further analyses were conducted on these data.

\section{Discussion}

$\nabla$

The goals of this research were to examine whether the cannabinoid constituents THC or CBD alone can attenuate or prevent CIN. The current study demonstrates that THC and CBD alone can produce an attenuation of cisplatin-induced tactile allodynia similar to that of gabapentin. These findings suggest that the use of a THC/CBD in combination like Sativex may not be necessary in treating CIN. To our knowledge, this is the first attempt to study CBD alone in a CIN murine model and show a potential clinical

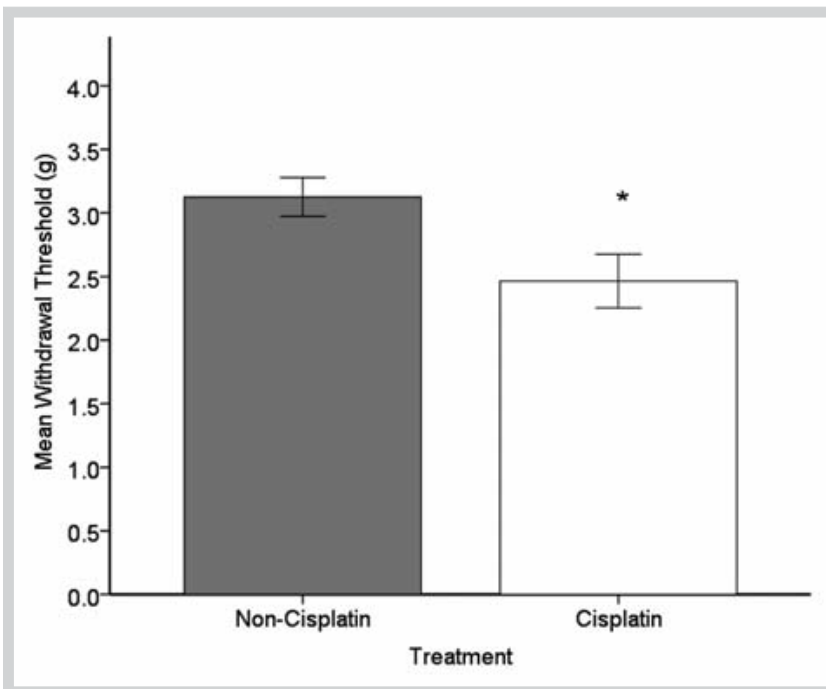

Fig. 1 Effects of 9 dosings of cisplatin across 18 days on tactile allodynia. Values represent the mean paw threshold \pm SE $(n=16-18)$. ${ }^{*}$ Denotes significant difference of withdrawal threshold compared to non-cisplatin group $(p<0.05)$.

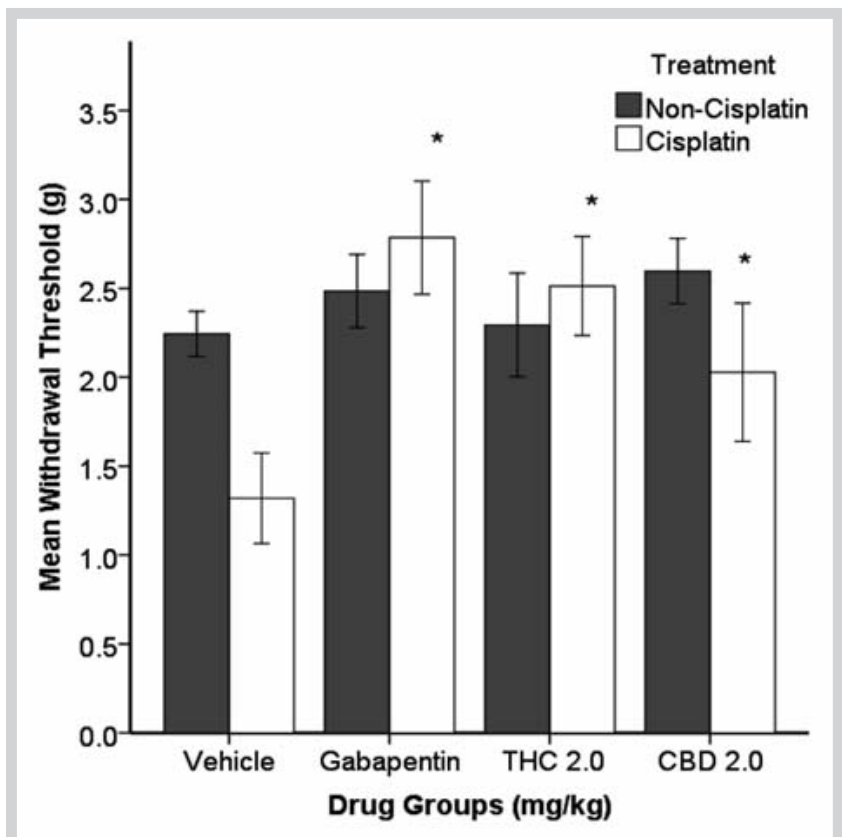

Fig. 2 Drug treatment effects of tactile allodynia on non-cisplatin and cisplatin mice. Values represent the mean paw threshold \pm SE $(n=4-5$ per group). * Denotes significant attenuation of tactile allodynia compared to the vehicle group $(p<0.05)$.

benefit of this cannabinoid constituent in an oncology setting. Unfortunately, our findings also indicate that neither THC nor CBD can prevent CIN when administered prophylactically.

Collectively, the current studies fit well with the existing literature on the role of cannabinoid modulation of CIN. For example, Vera et al. [12] demonstrated that cisplatin tactile allodynia in rats can be attenuated by administration of the $\mathrm{CB}$ selective and nonselective receptor agonists ACEA, JWH133, and WIN 55,212-2. 


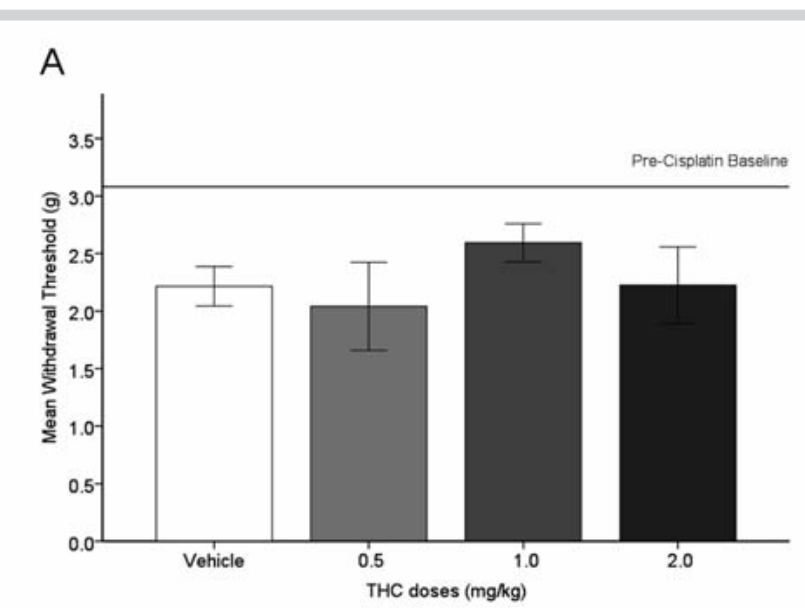

B

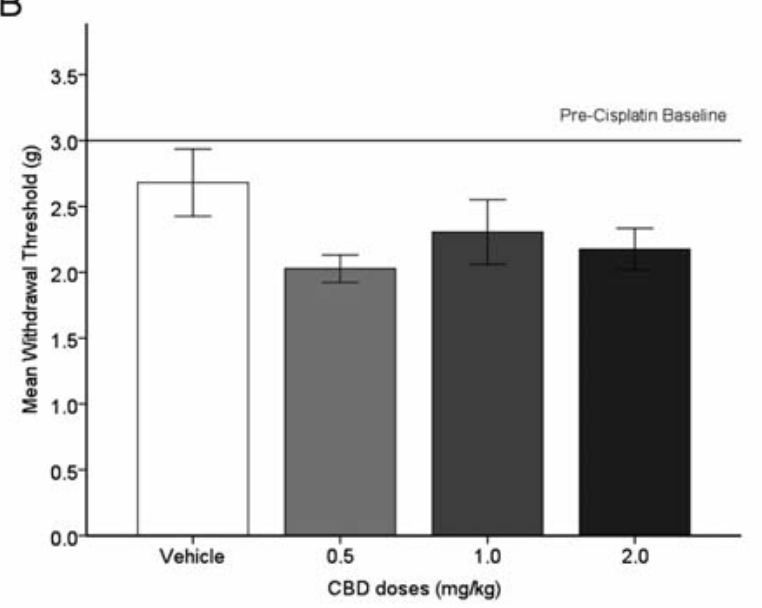

Fig. 3 A Effects of 6 dosings of saline and THC administered prior to cisplatin across 13 days on tactile allodynia. Values represent the mean paw withdrawal threshold \pm SE ( $n=3-4$ per group). B Effects of 6 dosings of saline and CBD administered prior to cisplatin across 13 days on tactile allodynia. Values represent the mean paw withdrawal threshold $\pm S E(n=3-4$ per group).

Cisplatin tactile allodynia can also be attenuated through increased levels of the endocannabinoids anandamide and 2arachidonylglycerol (2AG) via inhibition of fatty acid amide hydrolase (FAAH) and monoacylglycerol lipase (MGL), respectively [13].

While targeting the endocannabinoid system with THC and CBD can modulate CIN, there is a literature that reports THC exposure can lead to increased cancer cell proliferation and tumor metastasis by decreasing antitumor immune responses. Thus, the use of Sativex may be contra-indicated in treating CIN in oncology settings. Evidence suggests that these contradictory findings may reflect dose-dependent effects where macromolar concentrations of exogenous CBs have antitumor properties while clinically relevant micromolar doses have tumor promoting properties. This biphasic dose-effect is also observed with THC $[19,20]$. These data, along with the findings from the current study, suggest that CBD alone may be a viable alternative than THC or synthetic CB compounds and prove clinically efficacious in CIN in oncology settings.

\section{Materials and Methods}

$\nabla$

\section{Subjects}

Male C57BL/6 mice (25-30 g) were used in these studies. Food and water were provided ad libitum. Lights operated on a $12: 12$-h light-dark cycle with lights on at 06:00. Mice were housed two per polycarbonate tub with soft bedding. Mice used in Experiment 1 were experimentally naïve. A subset of mice that served as a control group (i.e., non-cisplatin) from Experiment 1 were enrolled in Experiment 2. These mice were given a 1-week drug washout before being enrolled in the cisplatin administration protocol. The following procedures were approved March 9, 2013 by the Institutional Animal Care and Use Committee at the University of Mississippi (Protocol \#13-017).

\section{Drugs}

Cisplatin (TSZ-Chemical; purity > 99\%) was dissolved in $0.85 \%$ saline to yield dosages of 2.3 and $1.0 \mathrm{mg} / \mathrm{kg} / \mathrm{mL}$ (see studies below). Lactated Ringer's solution (Fisher Scientific; $0.25 \mathrm{~mL}$ ) was used to hydrate the mice in order to prevent kidney and liver damage associated with repeated cisplatin administration. Gabapentin (TCI America; purity > 98\%) served as the reference compound and was dissolved in saline to yield a dose of $100 \mathrm{mg} / \mathrm{kg} /$ mL. CBD (0.5-2.0 mg/kg/mL; > 98\% purity) or THC (0.5-2.0 mg/ $\mathrm{kg} / \mathrm{mL}$; purity > 98\%; NIDA Drug Supply Program) was dissolved in $5 \%$ ethanol and 5\% cremophor in injectable water. These doses were selected because they did not produce motor impairments, whereas higher doses of THC did.

\section{Behavioral measures}

An eVF (Topcat Metrology Ltd.) was used to measure paw withdrawal thresholds to a mechanical stimulus and determined the presence of tactile allodynia in cisplatin-treated mice. Mice were acclimated in a rectangular enclosure $(3.81 \times 11.43 \times 11.43 \mathrm{~cm})$ with metal rod floors for at least 5 min before testing. A Von Frey filament was applied to the mid-plantar region of the hind paw and withdrawal thresholds were recorded. Filaments were applied to alternating left and right hind paws at 3 min intervals for a total of 4 measurements per paw. The average score of these eight tests served as the dependent measure.

Experiment 1 protocol: Mice were randomly assigned to receive 9 IP injections of either cisplatin $(2.3 \mathrm{mg} / \mathrm{kg} / \mathrm{mL})$ or saline every other day with lactated Ringer's solution $(1.0 \mathrm{~mL} / \mathrm{kg})$ on non-cisplatin days (Table 1S, Supporting Information). Baseline eVF measurements were taken on day 1 and revealed a balanced group assignment. To assess the presence of tactile allodynia, additional eVF measurements were taken on ringers days 3, 6, and 9 prior to administration. On ringers day 9, eVF measurements revealed that cisplatin mice showed significantly lower paw withdrawal thresholds (i.e., allodynia) than the controls. The following day, pair-housed mice were separated in their home tubs via a metal divider and block assigned into saline, gabapentin (100 mg/kg), THC (2 mg/kg), or CBD (2 mg/kg) groups ( $\mathrm{n}=4-5)$. The following 2 days the mice were given drugs via IP administration and assessed on eVF $1 \mathrm{~h}$ after administration. To accommodate eVF testing for all mice, drug screening was conducted across 2 days and counterbalanced across groups. Animals that lost $>25 \%$ of body weight were removed from the study and humanely euthanized ( 1 per experiment).

Experiments $2 a$ and $b$ protocol: In contrast to Experiment 1, mice received 6 IP injections of cisplatin (Exp 2a: $2.3 \mathrm{mg} / \mathrm{kg} / \mathrm{mL}$ or Exp 2b: $1.0 \mathrm{mg} / \mathrm{kg} / \mathrm{mL}$ ) every other day with lactated Ringer's solution 
on non-cisplatin days (Table $\mathbf{2} \mathbf{S}$, Supporting Information). We found significant tactile allodynia after 6 cisplatin dosings and that prompted a change from 9 dosings in Experiment 1 to 6 dosings in Experiment 2. In an attempt to reduce animal numbers requiring a humane endpoint but still achieve significant tactile allodynia, a literature suggested that we lower the dosage of cisplatin from $2.3 \mathrm{mg} / \mathrm{kg}$ to $1.0 \mathrm{mg} / \mathrm{kg}$. Separate cohorts of mice were randomly assigned into either CBD (Exp 2a: 0.5, 1.0, and $2.0 \mathrm{mg} / \mathrm{kg}$ ) or THC (Exp 2b: $0.5,1.0$, and $2.0 \mathrm{mg} / \mathrm{kg}$ ) treatment conditions. The cannabinoid vehicle served as a control group. Sample sizes were $n=3-4$. Baseline eVF measures taken on day 1 revealed a balanced group assignment. Cannabinoid test drugs were administered IP 30 min prior to cisplatin administration in attempts to prevent the development of allodynia associated with CIN. eVF measurements were collected prior to the administration of cisplatin 3,5, and the day following ringers 6 . Animals that lost $>25 \%$ of body weight were removed from the study and humanely euthanized ( 1 per experiment).

\section{Supporting information}

Order and time sequence of testing for Experiments 1 and 2 are available as Supporting Information.

\section{Acknowledgements}

$\nabla$

This study was supported by Grant Number P20GM104932 from the National Institute of General Medical Sciences (NIGMS), a component of the National Institutes of Health (NIH), and its contents are solely the responsibility of the authors and do not necessarily represent the official view of NIGMS or NIH.

\section{Conflict of Interest \\ $\nabla$}

The authors declare no conflict of interest.

\section{References}

1 Valeberg BT, Rustøen T, Bjordal K, Hanestad BR, Paul S, Miaskowski C. Self-reported prevalence, etiology, and characteristics of pain in oncology outpatients. Eur J Pain 2008; 12: 582-590

2 Kumar SP. Cancer pain: A critical review of mechanism-based classification and physical therapy management in palliative care. Indian J Palliat Care 2011; 17: 116-126

3 Grisold W, Cavaletti G, Windeback AJ. Peripheral neuropathies from chemotherapeutics and targeted agents: diagnosis, treatment, and prevention. Neuro Oncol 2012; 14: iv45-iv54
4 Windebank AJ, Grisold W. Chemotherapy-induced neuropathy. J Peripher Nerv Syst 2008; 13: 27-46

5 Seretny M, Currie GL, Sena ES, Ramnarine S, Grant R, MacLeod MR, Colvin LA, Fallon M. Incidence, prevalence and predictors of chemotherapy-induced peripheral neuropathy: A systematic review and meta-analysis. Pain 2014; 155: 2461-2470

6 Kannarkart G, Lasher EE, Schiff D. Neurologic complications of chemotherapy agents. Curr Opin Neurol 2007; 20: 719-725

7 Paice, JA Chronic treatment-related pain in cancer survivors. Pain 2010; 152: S84-S89

8 Wolf S, Barton D, Kottschade L, Grothery A, Loprinzi C. Chemotherapyinduced peripheral neuropathy: prevention and treatment strategies. Eur J Cancer 2008; 44: 1507-1515

9 Chiou LC, Hu SS, Ho Y. Targeting the cannabinoid system for pain relief? Acta Anaesthesiol Taiwan 2013; 51: 161-170

10 Rahn EJ, Thakur GA, Wood JAT, Zvonok AM, Makrynannis A, Hohmann AG. Pharmacological characterization of AM1710, a putative cannabinoid CB2 agonist from the cannbilactone class: antinociception without central nervous system side-effects. Pharmacol Biochem Behav 2011; 98: 493-502

11 Curto-Reyes $V$, Botok $T$, Hidalgo A, Menéndez L, Baamonde A Antinociceptive effects induced through the stimulation of spinal cannabinoid type 2 receptors in chronically inflamed mice. Eur J Pharmacol 2011; 668: 184-189

12 Vera G, Cabezos PA, Martín MI, Abalo R. Characterization of cannabinoid-induced relief of neuropathic pain in a rat model of cisplatininduced neuropathy. Pharmacol Biochem Behav 2013; 105: 205-212

13 Guindon J, Lai Y, Takacs SM, Bradshaw HB, Hohmann AG. Alterations in endocannabinoid tone following chemotherapy-induced peripheral neuropathy: effects of endocannabinoid deactivation inhibitors targeting fatty-acid amide hydrolase and monoacylglycerol lipase in comparison to reference analgesics following cisplatin treatment. Pharmacol Res 2013; 67: 94-109

14 Khasabova IA, Khasabov S, PazJ, Harding-Rose C, Simone DA, Seybold VS. Cannabinoid type-1 receptor reduces pain and neurotoxicity produced by chemotherapy. J Neurosci 2012; 32: 7091-7101

15 Alexander A, Smith PF, Rosengren RJ. Cannabinoids in the treatment of cancer. Cancer Lett 2009; 285: 6-12

$16 \mathrm{Booz}$ GW. Cannabidiol as an emergent therapeutic strategy for lessening the impact of inflammation on oxidative stress. Free Radic Biol Med 2011; 51: 1054-1061

17 Rahn EJ, Hohmann AG. Cannabinoids as pharmacotherapies for neuropathic pain: from the bench to the bedside. Neurotherapeutics 2009; 6: 713-737

18 Nurmikko TJ, Seprell MG, Hoggart B, Toomey PJ, Morlion BJ, Haines D. Sativex successfully treats neuropathic pain characterized by allodynia: a randomized, double-blind, placebo-controlled clinical trial. Pain 2007; 133: 210-220

19 Pisanti S, Picardi P, D'Alessandro A, Laezza C, Bifulco M. The endocannabinoid signaling system in cancer. Trends Pharmacol Sci 2013; 34: 273-282

20 Hart S, Fischer OM, Ullrich A. Cannabinoids induce cancer cell proliferation via tumor necrosis factor alpha-converting enzyme (TACE/ ADAM17)-mediated transactivation of the epidermal growth factor receptor. Cancer Res 2004; 64: 1943-1950 